\title{
Activation of the EGFR-PI3K-CaM pathway by PRL-1-overexpressing placenta-derived mesenchymal stem cells ameliorates liver cirrhosis via ER stress-dependent calcium
}

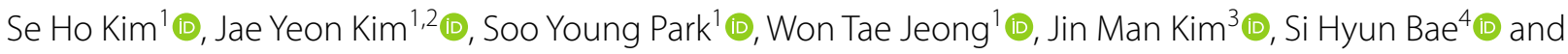
Gi Jin Kim ${ }^{1,2^{*}}$ (10)

\begin{abstract}
Background: Cholesterol accumulation and calcium depletion induce hepatic injury via the endoplasmic reticulum (ER) stress response. ER stress regulates the calcium imbalance between the ER and mitochondria. We previously reported that phosphatase of regenerating liver-1 (PRL-1)-overexpressing placenta-derived mesenchymal stem cells (PD-MSCs ${ }^{\text {PRL-1 }}$ ) promoted liver regeneration via mitochondrial dynamics in a cirrhotic rat model. However, the role of

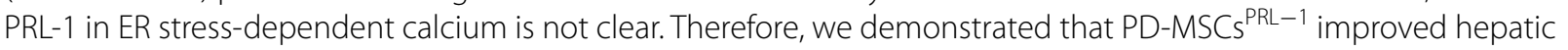
functions by regulating ER stress and calcium channels in a rat model of bile duct ligation (BDL).

Methods: Liver cirrhosis was induced in Sprague-Dawley (SD) rats using surgically induced BDL for 10 days. PDMSCs and PD-MSCs ${ }^{\text {PRL-1 }}\left(2 \times 10^{6}\right.$ cells) were intravenously administered to animals, and their therapeutic effects were analyzed. WB-F344 cells exposed to thapsigargin (TG) were cocultured with PD-MSCs or PD-MSCs ${ }^{\text {PRL-1. }}$.

Results: ER stress markers, e.g., eukaryotic translation initiation factor $2 a(e l F 2 a)$, activating transcription factor 4 (ATF4), and C/EBP homologous protein (CHOP), were increased in the nontransplantation group (NTX) compared to the control group. PD-MSCs ${ }^{\text {PRL-1 }}$ significantly decreased ER stress markers compared to NTx and induced dynamic changes in calcium channel markers, e.g., sarco/endoplasmic reticulum $\mathrm{Ca}^{2+}$-ATPase 2b (SERCA2b), inositol 1,4,5-trisphosphate receptor (IP3R), mitochondrial calcium uniporter (MCU), and voltage-dependent anion channel 1 (VDAC1) $\left({ }^{*} p<0.05\right)$. Cocultivation of TG-treated WB-F344 cells with PD-MSCS ${ }^{\text {PRL-1 }}$ decreased cytosolic calmodulin (CaM) expression and cytosolic and mitochondrial $\mathrm{Ca}^{2+}$ concentrations. However, the ER $\mathrm{Ca}^{2+}$ concentration was increased compared to PD-MSCs $\left({ }^{*} p<0.05\right)$. PRL-1 activated phosphatidylinositol-3-kinase (PI3K) signaling via epidermal growth factor receptor (EGFR), which resulted in calcium increase via CaM expression.
\end{abstract}

Conclusions: These findings suggest that PD-MSCs ${ }^{\text {PRL-1 }}$ improved hepatic functions via calcium changes and attenuated ER stress in a BDL-injured rat model. Therefore, these results provide useful data for the development of next-generation MSC-based stem cell therapy for regenerative medicine in chronic liver disease.

Keywords: Calcium homeostasis, ER stress, Liver cirrhosis, Liver regeneration, Mitochondria, Placenta-derived mesenchymal stem cells, Phosphatase of regenerating liver-1

*Correspondence: gjkim@cha.ac.kr

1 Department of Biomedical Science, CHA University, Seongnam 13488, Republic of Korea

Full list of author information is available at the end of the article permits use, sharing, adaptation, distribution and reproduction in any medium or format, as long as you give appropriate credit to the original author(s) and the source, provide a link to the Creative Commons licence, and indicate if changes were made. The images or other third party material in this article are included in the article's Creative Commons licence, unless indicated otherwise in a credit line to the material. If material is not included in the article's Creative Commons licence and your intended use is not permitted by statutory regulation or exceeds the permitted use, you will need to obtain permission directly from the copyright holder. To view a copy of this licence, visit http://creativecommons.org/licenses/by/4.0/. The Creative Commons Public Domain Dedication waiver (http://creativeco mmons.org/publicdomain/zero/1.0/) applies to the data made available in this article, unless otherwise stated in a credit line to the data. 


\section{Introduction}

The endoplasmic reticulum (ER) is responsible for various cellular activities, such as protein secretion, synthesis, maturation, translation and folding in eukaryotic cells, and it plays an important role in regulating calcium concentrations [1]. ER stress induces the accumulation of misfolded proteins or calcium depletion in the ER lumen, and these events result in progression to severe stages of several diseases, such as diabetes, obesity, and nonalcoholic fatty liver disease (NAFLD)/nonalcoholic steatohepatitis (NASH) [2]. ER stress in liver fibrosis results in the accumulation of unfolded proteins, very low-density lipoprotein (VLDL), inflammatory cytokines and oxidative stress, especially $\mathrm{Ca}^{2+}$ imbalances in the ER lumen [3]. ER stress dysregulates calcium pump channels in chronic liver diseases [4], calcium inflow controls sarco/ endoplasmic reticulum $\mathrm{Ca}^{2+}$ ATPase (SERCA2b), and calcium release regulates inositol trisphosphate receptor (IP3R), ryanodine receptor 2 (RyR2) and calcium sensor stromal interaction molecule 1 (STIM1) in the ER membrane [5]. SERCA activity is impaired in NAFLD, which leads to imbalanced calcium homeostasis under ER stress [6].

The unfolded protein response (UPR) and abnormal UPR pathway restore normal functioning of the cell and cell death in cases of irreversible disruption. PKR-like ER kinase (PERK) is a key factor of the UPR that induces apoptosis via the transcription factor C/EBP homologous protein $(\mathrm{CHOP})$ and phosphorylation of eukaryotic translation initiation factor 2 alpha (eIF2 $\alpha$ ) to upregulate the expression of activating transcription factor 4 (ATF4). CHOP-deleted mice showed reduced apoptotic and necrotic hepatocyte death via decreases in the expression of alpha-smooth muscle actin ( $\alpha$-SMA) and transforming growth factor beta-1 (TGF- $\beta 1$ ) [7]. However, the role of ER stress-dependent calcium influx in liver diseases, including cirrhosis, is not known.

$\mathrm{Ca}^{2+}$-mobilizing growth factors generate $\mathrm{Ca}^{2+}$ signals in hepatocytes via receptor tyrosine kinases (RTKs). RTKs are membrane proteins that bind to ligands, such as growth factors, cytokines, and hormones, in the plasma membrane. Several RTKs are expressed in the liver, including hepatocyte growth factor receptor (HGFR), vascular endothelial growth factor receptor (VEGFR), platelet-derived growth factor receptor (PDGF), and epidermal growth factor receptor (EGFR) [8]. EGFR is a key regulator in early inflammation and hepatocyte proliferation in hepatic disease [9]. The activation of EGFR in liver disease affects liver regeneration via the downstream signaling pathway Ras-Raf-MEK-ERK1/2, and it controls the phosphatidylinositol-3-kinase (PI3K)-Akt mechanism [10]. For activation of RTK-based signaling, several ligands bind their specific receptors to activate phospholipase C (PLC) or PI3K, which hydrolyze phosphatidylinositol-4-5-bisphosphate (PIP2) to generate diacylglycerol (DAG) and inositol 1,4,5-trisphosphate (IP3). ER-resident IP3R calcium channels in chronic liver diseases lead to elevated cytosolic and decreased ER calcium levels [11]. A previous report suggested that IGF-1 regulated spliced X-box binding protein 1 (sXBP1) stability, protein synthesis and $\mathrm{Ca}^{2+}$ storage in the ER, which protected beta cell ER stress-mediated cell death at the onset of type 1 and type 2 diabetes [12]. However, the RTKs that regulate $\mathrm{Ca}^{2+}$ signaling in cirrhotic rat liver are not known.

Phosphatase of regenerating liver-1 (PRL-1) is a member of a small class of the prenylated PTP family, and it was identified as an immediate early gene in the regenerating rat liver [13]. Increased PRL-1 also regulated the proliferation and migration of mesenchymal stem cells in a liver cirrhosis rat model [14], and it shows high homology between humans and rats/mice [15]. Wang et al. reported that PRL-1 localized to the ER during the mitotic cell cycle [16]. During ER stress, increased PRL-1 suppressed apoptosis and enhanced ER function in a p53-dependent manner in injured cells [17]. A dual specificity tyrosine targeted by PRL- 1 in the $\mathrm{Ca}^{2+}$ pathway was reported [18]. However, research on PRL-1 direct modulation of the $\mathrm{Ca}^{2+}$ pathway is lacking, and the binding of PRL-1 to RTK ligands in the plasma membrane is not known.

Our previous reports demonstrated that placentaderived MSC (PD-MSC) transplantation enhanced hepatic function via increased antifibrotic effects, proliferation, and autophagy in a chronic liver disease model [19]. PRL-1-overexpressing PD-MSCs $\left(\mathrm{PD}-\mathrm{MSCs}^{\mathrm{PRL}-1}\right.$ ) also enhanced hepatic functions by increasing mitochondrial functions and proliferative potential in a chronic liver disease model compared to naïve PD-MSCs [20]. However, the relationship of PD-MSC ${ }^{\mathrm{PRL}-1}$ transplantation to the calcium-induced ER stress mechanism in cirrhotic livers is not clear. Therefore, the present study investigated whether PDMSC ${ }^{\text {PRL-1 }}$ implantation induced $\mathrm{Ca}^{2+}$ influx in injured liver tissues in a rat model of bile duct ligation (BDL), whether it induced $\mathrm{Ca}^{2+}$ influx and how it correlated with ER stress. Finally, we evaluated the therapeutic mechanism of PD-MSC ${ }^{\mathrm{PRL}-1}$ modulation of ER stressdependent calcium via RTK signaling in a rat model of BDL.

\section{Materials and methods \\ Cell culture}

The institutional review board of CHA Bundang Hospital, Seoul, Republic of Korea, approved the collection and 
use of samples for research purposes (IRB 07-18). All participants provided written informed consent prior to sample collection. Placentas were extracted from women who were free of medical, obstetrical, and surgical complications and delivered at term ( $\geq 37$ gestational weeks). PD-MSCs were isolated as previously described [21] and cultured in $\alpha$-modified minimal essential medium ( $\alpha$-MEM; HyClone Logan, UT, USA) supplemented with $10 \%$ fetal bovine serum (FBS; Gibco, Carlsbad, CA, USA), $1 \%$ penicillin/streptomycin (P/S; Gibco), $1 \mu \mathrm{g} / \mathrm{mL}$ heparin (Sigma-Aldrich, St. Louis, MO, USA), and $25 \mathrm{ng} / \mathrm{mL}$ human fibroblast growth factor-4 (hFGF-4; Peprotech, Inc., Rocky Hill, NJ, USA). The PRL-1 plasmid vector was purchased from Origene (\#RG200435; Rockville, MD, USA). For overexpression of the PRL-1 gene, naive PDMSCs (passage $=7$ ) were transfected using the AMAXA nucleofector system (Lonza, Basel, Switzerland) according to the manufacturer's instructions as previously described [20]. After transfection for $24 \mathrm{~h}$, the cells were selected using $1.5 \mathrm{mg} / \mathrm{mL}$ neomycin. WB-F344 cells (rat liver epithelial cells) were cultured at $37{ }^{\circ} \mathrm{C}$ in $\alpha$-MEM supplemented with 10\% FBS (Gibco) and 1\% P/S (Gibco). All cells were maintained at $37{ }^{\circ} \mathrm{C}$ in a $5 \% \mathrm{CO}_{2}$ incubator.

\section{Animal models and transplantation of MSCs}

Seven-week-old male Sprague-Dawley rats (Orient Bio, Inc., Seongnam, Republic of Korea) were maintained in an air-conditioned animal facility. Rats were anesthetized via intraperitoneal injection using avertin (2,2,2-tribromoethanol; Sigma-Aldrich) and underwent common BDL to induce liver cirrhosis as previously described. Ten days after BDL induction [22], naïve PD-MSCs (PDMSCs; $n=20$ ) and PD-MSCs ${ }^{\text {PRL-1 }}$ (PD-MSCs ${ }^{\text {PRL-1; }}$; $n=20$ ) were stained using the PKH67 Fluorescent Cell Linker Kit (Sigma-Aldrich) and injected intravenously through the tail vein $\left(2 \times 10^{6}\right.$ cells/animal $)$ in the transplantation group. Non-transplanted rats (NTx; $n=20)$ and sham control rats (Con; $n=5$ ) were maintained. The rats were sacrificed after $1,2,3$, and 5 weeks to extract liver tissues and blood samples. The Institutional Animal Care Use Committee of CHA University, Seongnam, Republic of Korea, approved the experimental processes and protocols for animal modeling (IACUC-200033).

\section{Liver histology and serum biochemistry}

Liver tissue samples were fixed in $10 \%$ neutral buffered formalin (NBF), embedded in paraffin, and sectioned at $5 \mu \mathrm{m}$ thickness for hematoxylin and eosin (H\&E) and Sirius red staining. Representative images of whole sections of the liver were captured and quantified using a digital slide scanner (3DHISTECH, Ltd., Budapest, Hungary). The serum concentrations of alanine aminotransferase
(ALT), aspartate aminotransferase (AST), total bilirubin (TBIL), and albumin (ALB) were measured from individual blood samples (Southeast Medi-Chem Institute, Busan, Republic of Korea).

\section{Immunofluorescence}

For analysis of gene expression in liver tissues, samples were sectioned at $6 \mu \mathrm{m}$ thickness and fixed using $4 \%$ paraformaldehyde. Liver tissues from each group were blocked using Protein Block Serum-Free solution (Dako, Santa Clara, CA, USA) for $1 \mathrm{~h}$. The following primary antibody was used: SERCA2b (1:1000; Invitrogen, Carlsbad, CA, USA). The sections were stained with 4',6-diamidino-2-phenylindole (DAPI; Invitrogen) and observed using confocal microscopy (LSM 700). Representative images were analyzed using ZEN blue software (Zeiss). The experiment was performed at least in duplicate.

\section{Immunohistochemistry}

To determine calcium channel expression in the ER, liver tissues from each group were sectioned at a thickness of $5 \mu \mathrm{m}$ and fixed with $10 \%$ NBF. The fixed tissues were reacted in $3 \%$ hydrogen peroxide $\left(\mathrm{H}_{2} \mathrm{O}_{2}\right)$ in $100 \%$ methanol to block endogenous peroxidase activity. The following antibodies were used: anti-CHOP (1:50; Santa Cruz, Dallas, TX, USA), anti-calmodulin (1:100; Novus Biologicals, Littleton, CO, USA) and anti-PCNA (1:500; company). CHOP was performed using Proteinase $\mathrm{K}$ $(20 \mu \mathrm{g} / \mathrm{mL})$ (Dako). However, anti-calmodulin and antiPCNA were used for antigen retrieval before incubation at $4{ }^{\circ} \mathrm{C}$ overnight. Incubation with horseradish peroxidase-conjugated streptavidin-biotin complex (Dako) and 3,3-diaminobenzidine (EnVision ${ }^{\mathrm{TM}}$ Systems, Santa Clara, CA, USA) was performed to generate a chromatic signal. For counterstaining, Mayer's hematoxylin (Dako) was used. Representative images were captured and quantified using a digital slide scanner (3DHISTECH, Ltd.).

\section{Quantitative real-time PCR}

Total RNA was isolated from samples using TRIzol (Invitrogen). cDNA was synthesized using Superscript III reverse transcription (Invitrogen) according to the manufacturer's instructions. For determinations of calcium channel factor expression in liver tissues and hepatocytes, qRT-PCR was performed using rat primers (Table 1, designed by BIONEER, Daejeon, Korea) and SYBR Green Master Mix (Roche, Basel, Switzerland) using the CFX Connect ${ }^{\mathrm{TM}}$ Real Time System (Bio-Rad, Hercules, CA, USA). Rat GAPDH was used as an internal control for normalization, and each sample was analyzed in duplicate. 


\section{Western blotting}

Homogenized liver tissues and samples were lysed in RIPA buffer containing a protease inhibitor cocktail (Roche) and phosphatase inhibitor (Sigma-Aldrich). Quantified protein extracts $(40 \mu \mathrm{g})$ were loaded in $6 \sim 15 \%$ sodium dodecyl sulfate polyacrylamide gel electrophoresis (SDS-PAGE). The separated proteins were transferred to polyvinylidene difluoride (PVDF) membranes (Bio-Rad). The following primary antibodies were used: anti-SERCA2b (1:500; Invitrogen), anti-mitochondrial calcium uniporter (MCU) (1:1000; Invitrogen), anti-IP3R (1:800; Cell Signaling Technology), anti-voltage-dependent anion-sensitive channel 1 (VDAC1), anti-total-eukaryotic initiation factor $2 \alpha$ (eIF $2 \alpha)$, antiphospho-eIF2 $\alpha$, anti-activating transcription factor 4 (ATF4), anti-CHOP, anti-PI3K-p110 $\alpha$ (1:1000; all from Cell Signaling Technology), anti-PI3K-p85 (1:3000; BD Biosciences, San Jose, CA, USA), anti-PERK (1:200; Santa Cruz), anti-GRP75 (1:500; Abcam), and anti-calmodulin (1:500; Novus Biologicals). The loading control was antiGAPDH (1:2000; AbFrontier, Seoul, Republic of Korea). After the membranes were washed, the following secondary antibodies were used: anti-mouse IgG and anti-rabbit IgG (1:8,000; all from Bio-Rad). The protein bands were detected using a Clarity Western ECL kit (Bio-Rad) and a ChemiDoc imaging system (Bio-Rad).

\section{Calcium influx using a fluorescence resonance energy transfer (FRET) biosensor}

For confirmation of calcium influx in hepatocytes, cytoplasm-targeting CMV-Y-GECO1, ER-targeting CMV-ER-LAR-GECO1, and mitochondria-targeting CMV-mito-GEM-GECO1 were purchased (Addgene, Watertown, MA, USA). Cells were transfected using
Lipofectamine 2000 (Thermo Fisher Scientific) for $4 \mathrm{~h}$ and treated with TG (500 nM) for $24 \mathrm{~h}$. For simultaneous monitoring of the $\mathrm{Ca}^{2+}$ concentration in the ER, cytoplasm, and mitochondria, widefield imaging was performed using a confocal microscope (LSM880; Carl Zeiss) at $37^{\circ} \mathrm{C}$ in a humidified atmosphere with $5 \% \mathrm{CO}_{2}$.

\section{Phospho-RTK proteome profiler array}

For analysis of the interaction of PRL-1 and RTKs, a human phospho-RTK Array kit (R\&D Systems, Minneapolis, USA) was used following the manufacturer's assay procedures. Briefly, cell lysates $\left(1 \times 10^{7}\right.$ cells $)$ were diluted and incubated overnight with an anti-phospho-tyrosineHRP detection antibody. Captured signals represented the amount of phosphorylated protein.

\section{Statistical analysis}

All experiments were performed in duplicate or triplicate. The results are presented as the means \pm standard deviation (SD). Student's $t$ test was performed for groupwise comparisons, and a $p$-value less than 0.05 was considered statistically significant. One-way ANOVA was performed for pairwise comparisons and multiple paired $\mathrm{t}$ tests.

\section{Results}

PD-MSCs ${ }^{\text {PRL-1 }}$ decreased ER stress in a rat model of BDL and hepatocytes

PERK-eIF2 $\alpha$-ATF4-CHOP signaling in the UPR pathway is implicated in liver diseases [23]. To analyze UPR pathway activation by $\mathrm{PD}-\mathrm{MSC} \mathrm{CRL}^{\mathrm{PR}-1}$ transplantation, we assessed the expression of CHOP, which is an ER stress-mediated transcription factor, in liver tissues using immunohistochemistry (IHC) (Fig. 1a). The expression

Table 1 Primer Sequences (Reverse Transcription Polymerase Chain Reaction)

\begin{tabular}{|c|c|c|c|c|}
\hline Type & Gene & Sequence $\left(5^{\prime}-3^{\prime}\right)$ & $\operatorname{Tm}\left({ }^{\circ} \mathrm{C}\right)$ & Accession no \\
\hline \multirow[t]{7}{*}{ Calcium channel } & SERCA2b & $\begin{array}{l}\text { F: 5'- CCAGTCGATTCTTACAGGTG -3' } \\
\text { R: 5'- GCGATGTTTGTGCCAGAAAA -3' }\end{array}$ & 56 & NM_001110823.2 \\
\hline & IP3R & $\begin{array}{l}\text { F: 5'- GGAGCCTCTGGTGAAAACGA -3' } \\
\text { R: 5'- CACCCATGTTCCTCAGCAGT -3' }\end{array}$ & 59 & NM_001007235.2 \\
\hline & GRP75 & $\begin{array}{l}\text { F: 5'- TGATGCCAATGGGATTGTGC -3' } \\
\text { R: 5'- CTGCTTCAACACGTTCCTTC -3' }\end{array}$ & 58 & NM_001100658.2 \\
\hline & CaM & $\begin{array}{l}\text { F: 5'- GTGAGGCATTCCGAGTCTTT -3' } \\
\text { R: 5'- TCATCTGTTAGCTTTTCCCCG -3' }\end{array}$ & 57 & NM_031969.3 \\
\hline & STIM1 & $\begin{array}{l}\text { F: 5'- ACAAGCTTATCAGCGTGGAG -3' } \\
\text { R: 5'- ACTTCCGGAAAGTCTCCTCA -3' }\end{array}$ & 57 & NM_001108496.2 \\
\hline & VDAC1 & $\begin{array}{l}\text { F: 5'- AACAGTAACACTCGCTTTGG -3' } \\
\text { R: 5'- TTGACGTTCTTGCCATCCAG -3' }\end{array}$ & 57 & NM_031353.2 \\
\hline & $\mathrm{MCU}$ & $\begin{array}{l}\text { F: 5'- GGGGTGTTTCTCCGACAACT -3' } \\
\text { R: 5'- GGAGGTCTCTCTTTGGTGGC -3' }\end{array}$ & 59 & NM_001106398.1 \\
\hline Internal control & GAPDH & $\begin{array}{l}\text { F: 5'- TCCCTCAAGATTGTCAGCAA -3' } \\
\text { R: 5'- AGATCCACAACGGATACATT - } 3^{\prime}\end{array}$ & 58 & NM_017008.4 \\
\hline
\end{tabular}


of CHOP in the nuclei of hepatocytes was significantly increased in the BDL-induced NTx group compared to the normal control (Con) group. Notably, the CHOP level was reduced in PD-MSCs ${ }^{\mathrm{PRL}-1}$, and $\mathrm{CHOP}$ translocation into the nucleus was significantly decreased (Fig. 1b, " $p<0.05$ ).

Phosphorylated eIF2 $\alpha$ (p-eIF2 $\alpha$ ) expression was increased at 1 and 2 weeks in the NTx group, but the expression of PERK, ATF4 and CHOP decreased (Fig. 1c). In contrast, the transplantation groups (Tx) showed decreased levels compared to the NTx group. Notably, the expression of PERK and p-eIF2 $\alpha$ was significantly reduced in the PD-MSC ${ }^{\text {PRL-1 }}$ group (Fig. $1 \mathrm{~d}-\mathrm{g}$, " $p<0.05$ ). Thapsigargin (TG) is an inhibitor of SERCA2b in hepatocyte-specific calcium channels on the ER membrane, and it resulted in cellular apoptosis (Fig. 1h). To induce ER stress in hepatocytes, we analyzed the expression of ER stress markers after $500 \mathrm{nM}$ TG treatment for $24 \mathrm{~h}$ regardless of PD-MSC and PD-MSC ${ }^{\text {PRL-1 }}$ cocultivation (Fig. 1i). The protein levels of ER stress markers in WB-F344 cells treated with TG were substantially increased. However, their expression levels were significantly decreased after PD-MSC cocultivation. PD-MSC ${ }^{\mathrm{PRL}-1}$ cocultivation resulted in significantly reduced expression compared to naïve PD-MSCs (Fig. 1j, $" p<0.05)$. These findings indicated that PD-MSCs ${ }^{\mathrm{PRL}-1}$ more efficiently decreased ER stress than naïve PD-MSCs in cirrhotic rat livers and hepatocytes.

\section{PD-MSCs ${ }^{\text {PRL-1 }}$ regulated calcium channels in a rat model of $B D L$ and hepatocytes}

A calcium imbalance between the ER and mitochondria in the liver leads to the development of chronic metabolic diseases and impaired organelle function [24]. Therefore, we confirmed the expression levels of calcium channels in the ER membrane (e.g., SERCA2b and IP3R) and mitochondria (e.g., VDAC1 and MCU) and ER-mitochondrial $\mathrm{Ca}^{2+}$ transfer factors (e.g., GRP75) in a rat model of BDL using Western blotting (Fig. 2a). The expression levels of SERCA2b, IP3R, and GRP75 were increased in the NTx group compared to the normal group. (Fig. $2 \mathrm{~b}-\mathrm{d},{ }^{*} p<0.05$ ). In contrast, all Tx groups showed decreased levels compared to the NTx group. GRP75 levels were significantly decreased in the PD-MSC ${ }^{\text {PRL-1 }}$ group at 1 and 3 weeks. However, IP3R and GRP75 expression in the PD-MSC ${ }^{\text {PRL-1 }}$ group was dramatically increased compared to the PDMSC group (Additional file 1: Figs. S2a and b). Notably, the mRNA and protein levels of VDAC1 and MCU were increased in the PD-MSC ${ }^{\text {PRL-1 }}$ Tx group (Fig. $2 \mathrm{e}$ and $\mathrm{f}$, Additional file 1: Fig. S2c and d, " $p<0.05)$. TG treatment induced a decrease in the expression levels of IP3R, VDAC1, and MCU, and the expression levels of GRP75 and CaM were increased in the WB-F344 hepatocyte cell line. PD-MSC ${ }^{\text {PRL-1 }}$ cocultivation significantly altered the expression levels of IP3R, VDAC1, MCU, GRP75, and CaM compared to naïve PD-MSCs, but no significant change was found in the mRNA levels between the treated group and the cocultivation group (Additional file 1: Fig. $\mathrm{S} 2 \mathrm{e}-\mathrm{i},{ }^{*} p<0.05$ ). However, protein levels were decreased (Fig. $2 \mathrm{~g}$ and h, * $p<0.05)$. We also confirmed the degree of colocalization with SERCA2b and ER Tracker using immunofluorescence (IF) staining (Fig. 2i). The expression of SERCA2b in the NTx group was increased, but the expression was significantly decreased in the PDMSC $^{\text {PRL-1 }}$ group compared to the NTx group and the PD-MSC group (Fig. 2j, " $p<0.05$ ). These results indicated that PD-MSCs ${ }^{\text {PRL-1 }}$ efficiently regulated calcium channels between the ER and mitochondria in cirrhotic rat livers and hepatocytes.

\section{PD-MSCs ${ }^{\text {PRL-1 }}$ regulated calcium influx in rat hepatocytes}

To further analyze the concentration of calcium in hepatocytes, we confirmed the mRNA and protein levels of SERCA2b using qRT-PCR and Western blotting, respectively. The mRNA expression levels were increased in the TG-treated group compared to the normal group but decreased in the cocultivation group (Fig. 3a). Although TG treatment reduced the mRNA expression, the protein expression of SERCA2b was significantly increased in cocultured PD-MSCs and PD-MSCs ${ }^{\text {PRL-1 }}$ (Fig. $3 \mathrm{~b}$ and $\left.c,{ }^{*} p<05\right)$. The mRNA expression of SERCA2b and STIM1, which are calcium sensors in the ER, was significantly increased in PD-MSCs ${ }^{\mathrm{PRL}-1}$ (Additional file 1 : Fig. S3a and b). To investigate the change in calcium in the ER, cytoplasm, and mitochondria of hepatocytes, we analyzed these organelles using calcium biosensors, including ER-LAR-GECO for the ER, Y-GECO1 for the cytoplasm, and mito-GEM-GECO1 for the mitochondria (Fig. 3d). The influx of ER $\mathrm{Ca}^{2+}$ in the TG-treated WB-F344 cells decreased, buts the influx of cytoplasmic and mitochondrial $\mathrm{Ca}^{2+}$ was enhanced. Notably, PDMSC $^{\mathrm{PRL}-1}$ cocultivation induced an enhanced ER $\mathrm{Ca}^{2+}$ concentration but decreased the influx of cytoplasmic and mitochondrial $\mathrm{Ca}^{2+}$ compared to PD-MSCs (Fig. 3e$\mathrm{g}$, $" p<0.05)$. These results indicated that PD-MSC ${ }^{\mathrm{PRL}-1}$ cocultivation modulated calcium influx in ER stressinduced hepatocytes.

\section{PRL-1 regulated EGFR-PI3K-CaM calcium signaling in the BDL-injured rat liver}

To further investigate PRL-1 calcium signaling, we analyzed the expression of EGFR in hepatocytes injured by 


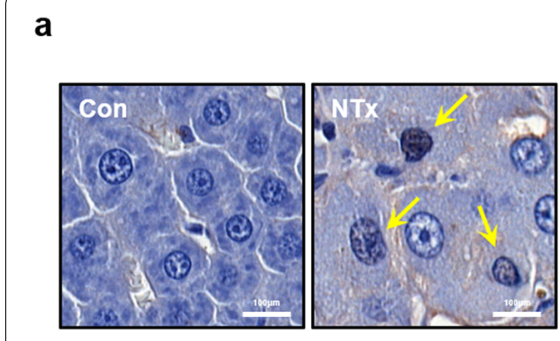

C

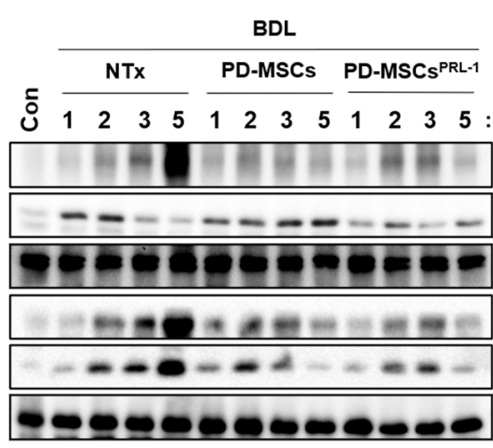

h

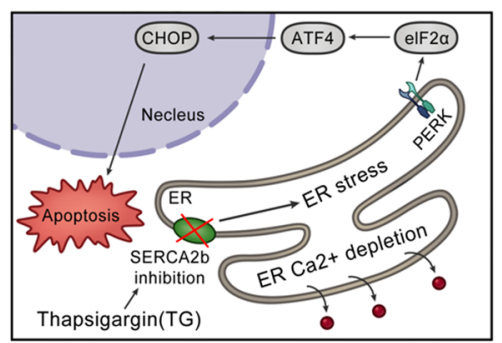

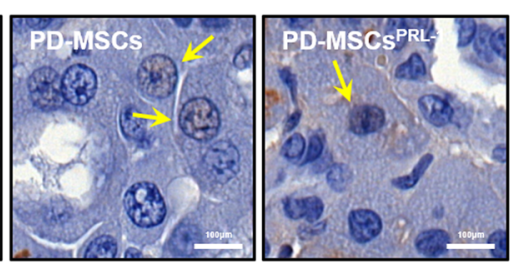

d

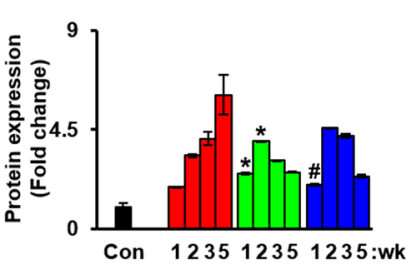

$$
\text { f }
$$
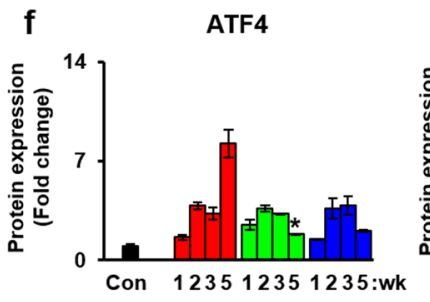

b

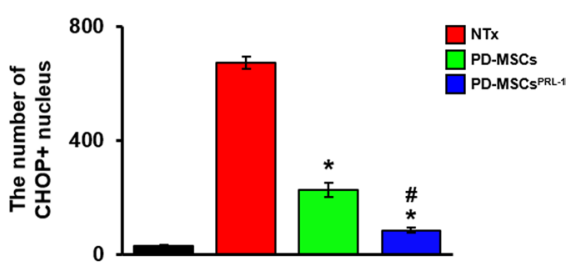

e p-elF2 $\alpha \quad \square^{\mathrm{NTx}}$
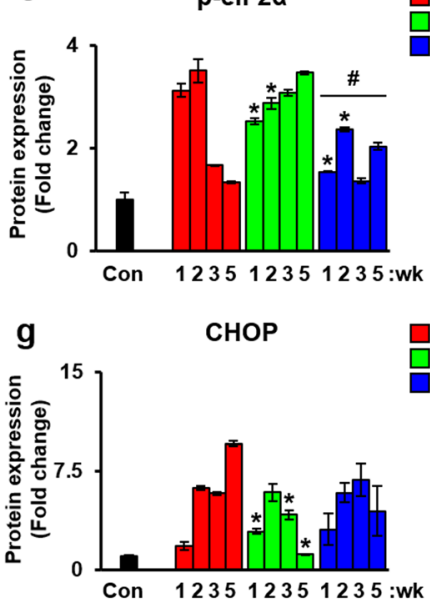

PD-MSCs PD-MSCs ${ }^{\text {PRL-1 }}$

i

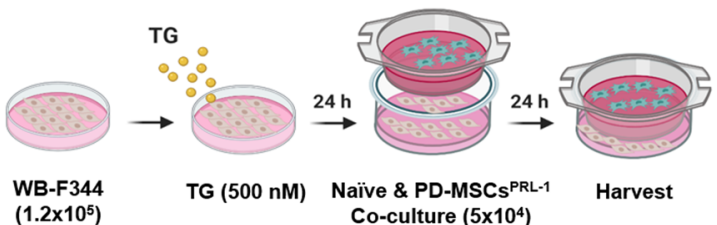

j

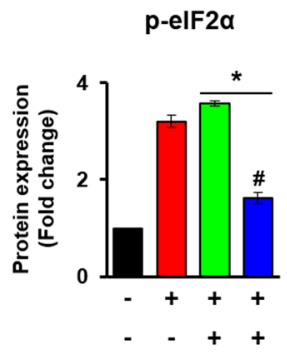

ATF4

CHOP

口PD-Mscs PD-MSCs ${ }^{\text {PQL-1 }}$
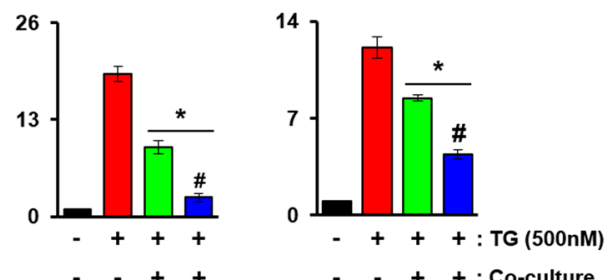

Fig. 1 PD-MSC ${ }^{P R L-1}$ transplantation decreased ER stress in a BDL rat model and hepatocytes. a Representative IF images of CHOP in cirrhotic livers at 5 weeks using IHC. $\mathbf{b}$ The number of CHOP-positive cells translocated into the nucleus in a cirrhotic liver. c Western blotting of ER stress markers (e.g., PERK, elF2a, ATF4, and CHOP) in a rat BDL. d-g Quantification of ER stress markers (e.g., PERK, total and phosphorylated elF2 alpha; p-elF2a, ATF4, and CHOP) following PD-MSC ${ }^{\text {PRL-1 }}$ transplantation in pooled BDL-injured rat liver protein ( $n=5 /$ group) at $1,2,3$, and 5 weeks. $\mathbf{h ~ A ~}$ schematic diagram showing TG-induced ER stress. i A schematic diagram describing TG (500 nM)-treated WB-F344 cells cocultured with PD-MSCs or PD-MSC ${ }^{\text {PRL-1 }}$ for $24 \mathrm{~h}$. j Western blotting and the intensities of ER stress markers in WB-F344 cells. a-tubulin was used as a loading control. Data from each group are shown as the means \pm SD and were assessed using Student's t test. ${ }^{*} p<0.05$ vs. NTx, \#p<0.05 vs. PD-MSCs. ATF4, activating transcription factor 4; BDL, bile duct ligation; CHOP, C/EBP homologous protein; $\mathrm{p}$-elF2a, phosphorylated elF2 alpha; PRL-1, phosphatase of regenerating liver-1; TG, thapsigargin

pentamidine regardless of cocultivation and recombinant PRL-1 treatment. As shown in Fig. 4a, PRL-1 binds to EGFR and activates PI3K-p110 $\alpha$ expression. PIP2 is divided into IP3, and the released IP3 binds to IP3R on the
ER membrane. These signaling pathways release $\mathrm{Ca}^{2+}$ and regulate the cellular response via the $\mathrm{Ca}^{2+} / \mathrm{CaM}$ complex (Fig. 4a). We performed an RTK dot blot assay and identified which of the 49 different RTKs were phosphorylated 


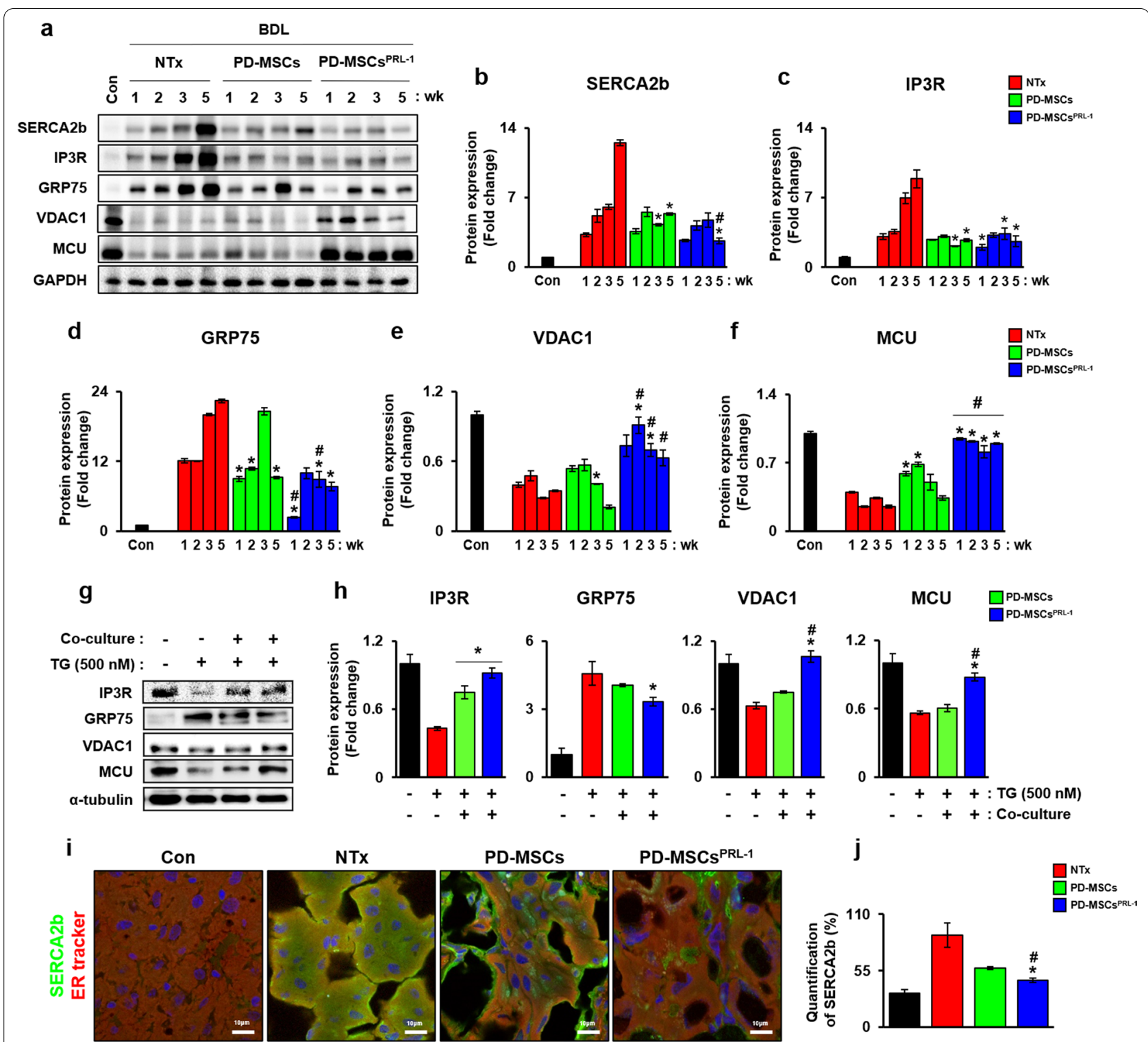

Fig. 2 PD-MSC ${ }^{P R L-1}$ transplantation regulated calcium channels in a rat model of BDL and hepatocytes. a Western blotting and $\mathbf{b}$-f the intensities of calcium channels (e.g., SERCA2b, IP3R, glucose regulated protein 75; GRP75, voltage-dependent anion channel 1; VDAC1, and mitochondria calcium uniporter; MCU) following PD-MSC ${ }^{\text {PRL-1 }}$ transplantation in BDL-induced rat liver protein measured using glycraldehyde-3-phosphate dehydrogenase (GAPDH) as a loading control. $\mathbf{g}$ Western blotting and $\mathbf{h}$ the intensities of calcium channels (e.g., IP3R, GRP75, VDAC1, MCU, and CaM) in TG (500 nM)-treated WB-F344 cells cocultured with PD-MSCs or PD-MSCs PRL-1 for $24 \mathrm{~h}$. a-Tubulin was used as a loading control. i Representative images and $\mathbf{j}$ quantification of SERCA2b (green) and ER Tracker (red) in BDL-injured rat livers at 5 weeks using IF staining. DAPI (blue) was used for counterstaining. Data from each group are shown as the means \pm SD and were assessed using Student's t test. ${ }^{*} p<0.05$ vs. NTx, \#p < 0.05 vs. PD-MSCs. BDL, bile duct ligation; CaM, calmodulin; GAPDH, glycraldehyde-3-phosphate dehydrogenase; GRP75, glucose regulated protein 75; IP3R, inositol trisphosphate receptor; $M C U$, mitochondrial calcium uniporter; NTx, nontransplantation; PRL-1, phosphatase of regenerating liver-1; SERCA2b, sarco/endoplasmic reticulum Ca ${ }^{2+}$ ATPase; VDAC1, voltage-dependent anion channel 1

(Fig. 4b). Compared to PD-MSCs, PD-MSCs ${ }^{\text {PRL-1 }}$ strongly activated phosphorylated EGFR after recombinant PRL-1 treatment. Recombinant PRL-1 and pentamidine inhibition treatment reduced the activity of phosphorylated EGFR (Fig. 4c, " $p<0.05$ ). The protein and mRNA expression of PI3K, which is a downstream factor of EGFR through
PRL-1, was increased in the PD-MSC ${ }^{\mathrm{PRL}-1}$ group compared to the NTx and PD-MSC groups, but the CaM levels were strongly decreased at 1,3 , and 5 weeks (Fig. $4 \mathrm{~d}-\mathrm{f}$ and Additional file 1: Fig. S4c-e, " $p<0.05)$. Endogenous CaM levels in cirrhotic livers in the NTx group were significantly increased, but its expression levels were significantly 


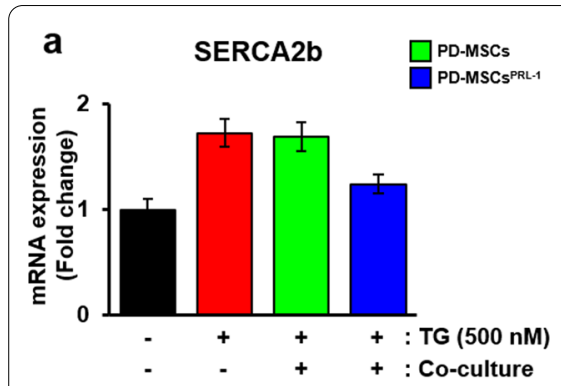

d
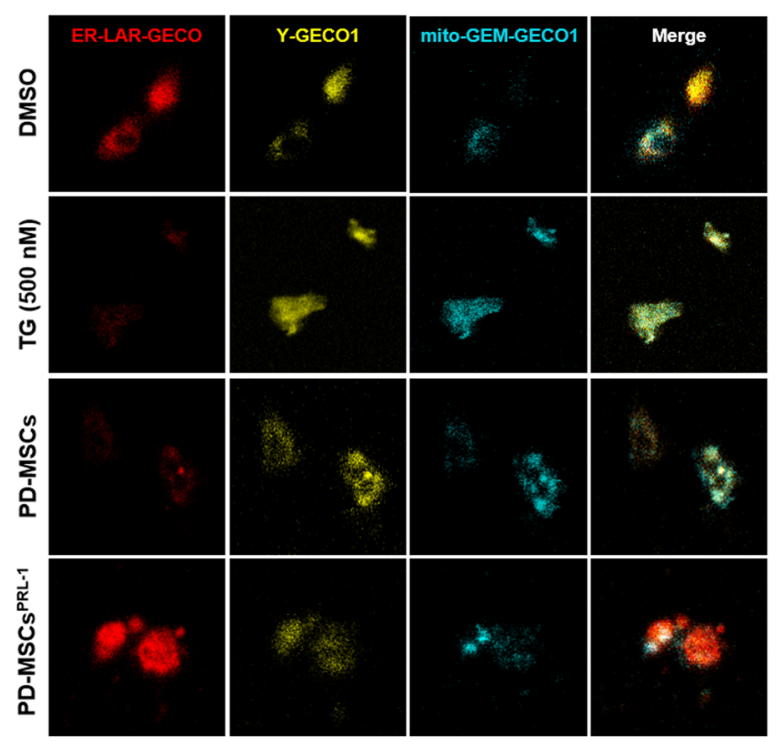
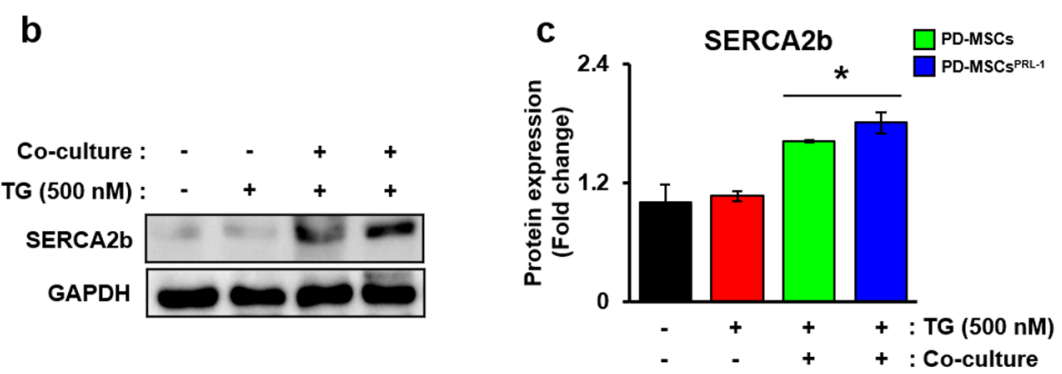

e

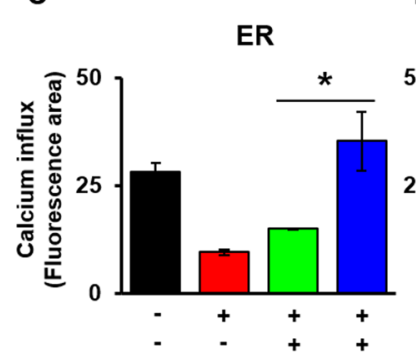

f

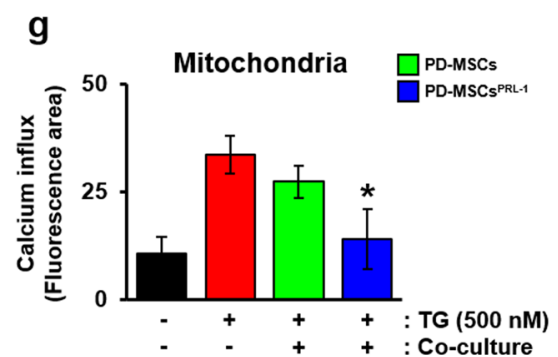

Fig. 3 PD-MSCs ${ }^{\text {PRL-1 }}$ regulated calcium influx in rat hepatocytes. a mRNA and $\mathbf{b}$, c protein levels of SERCA2b in TG (500 nM)-treated WB-F344 cells cocultured with PD-MSCs or PD-MSCS ${ }^{\text {PRL-1 }}$ for $24 \mathrm{~h}$. GAPDH was used as a loading control. $\mathbf{d}$ Representative fluorescence images and $\mathbf{e}-\mathbf{g}$ the fluorescence intensities of calcium influx using ER-LAR GEGO for ER (red), Y-GECO1 for cytoplasm (yellow), and mt-GEM-GECO1 for mitochondria (cyan blue) in TG (500 nM)-treated WB-F344 cells cocultured with PD-MSCs or PD-MSCs ${ }^{\text {PRL-1 }}$ for $24 \mathrm{~h}$. Data from each group are shown as the means $\pm S D$ and were assessed using Student's t test. ${ }^{*} p<0.05$ vs. NTx, \#p<0.05 vs. PD-MSCs. GAPDH, glycraldehyde-3-phosphate dehydrogenase; NTx, nontransplantation; PRL-1, phosphatase of regenerating liver-1; SERCA2b, sarco/endoplasmic reticulum Ca ${ }^{2+}$ ATPase; TG, thapsigargin

reduced in the PD-MSC ${ }^{\mathrm{PRL}-1}$ group compared to the naïve PD-MSC group (Fig. $4 \mathrm{~g}$ and $\mathrm{h},{ }^{*} p<0.05$ ). These results suggest that PRL-1 interacts with EGFR-PI3K-CaM and regulates calcium levels in BDL-injured rat livers.

\section{Hepatic regenerative effects of PD-MSCs ${ }^{\text {PRL-1 }}$ in a rat model of BDL}

We previously reported that the administration of PDMSCs $^{\text {PRL-1 }}$ improved hepatic functions in a rat model of BDL [20]. Collagen accumulation in liver tissues of the NTx group was significantly increased compared to the normal group. However, the number of Sirius red-positive areas significantly decreased in all $\mathrm{Tx}$ groups. Notably, antifibrotic effects due to decreased collagen deposition were dramatically observed in the PD-MSC ${ }^{\text {PRL-1 }}$ group compared to the PD-MSC group (Fig. 5a and b, $" p<0.05$ ). Blood chemistry analysis revealed that the levels of ALT, AST, and TBIL were decreased in the transplantation groups compared to the NTx group, and the ALB level was significantly increased (Fig. $5 \mathrm{c}-\mathrm{f},{ }^{*} p<0.05$ ). The PD-MSC ${ }^{\mathrm{PRL}-1}$ group exhibited substantially decreased ALT and AST and increased ALB levels. To further confirm the proliferation of hepatocytes in the livers of BDL-induced rats following transplantation, we examined proliferating cell nuclear antigen (PCNA) using IHC (Fig. 5g). Compared to naïve PD-MSCs, the positive signal was significantly enhanced in the PD-MSC ${ }^{\mathrm{PRL}-1}$ groups (Fig. 5h, " $p<0.05$ ). The expression of hepatic ALB and proliferation markers (e.g., CDK4 and cyclin D1) in the PD-MSC ${ }^{\mathrm{PRL}-1}$ group was remarkably higher than the PD-MSC group (Figs. 5i, 6). The correlation coefficient between ALB and cyclin D1 expression was $R^{2}=0.8819$ (Fig. 5j). These results suggested that 


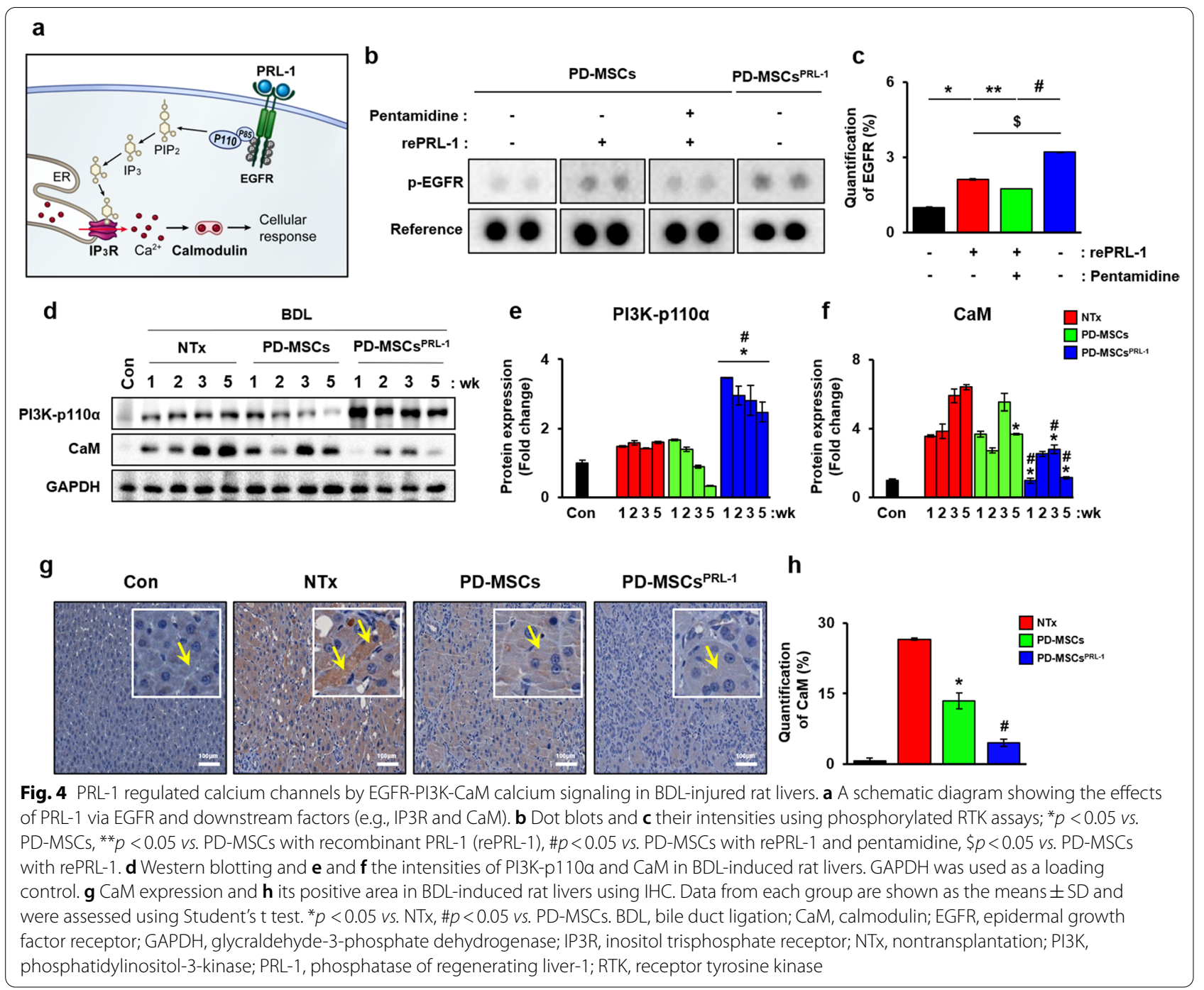

PD-MSCs ${ }^{\text {PRL-1 }}$ promoted hepatic regeneration in a BDL-injured rat model.

\section{Discussion}

Abnormal calcium levels lead to the ER stress response, which contributes to hepatic lipid accumulation and apoptosis in chronic liver disease [25]. This ER stress response is perturbed due to a calcium depletion-induced accumulation of unfolded/misfolded proteins [26]. The PERK-eIF2 $\alpha$-ATF4 axis mediates the pharmacological ER stress-induced hepatosteatosis that results from lipid homeostasis via several pathways [27]. Therefore, PERK deficiency and increased reactive oxygen species (ROS), due to accumulated lipids and protein aggregation, induce apoptosis in response to ER stress and eIF2 $\alpha$ phosphorylation [1]. AMPK inhibition and triglyceride accumulation stimulated hepatic steatosis in mice with liver-specific ATF4 knockout [28]. Increased phosphorylated PERK and CHOP levels also induced intracellular $\mathrm{Ca}^{2+}$ overload via the upregulation of Bax expression and increase in the active form of caspase-3 [29]. Therefore, persistent ER stress causes several degenerative diseases, including liver diseases associated with steatosis, via cell death [1]. Many scientists recently reported the efficacies of MSCs on chronic diseases, such as diabetic lung fibrosis, hepatic steatosis and chromium intoxication, by decreasing ER stress [30]. MSCs reduced the ER stress response by decreasing XBP1 and Bip expression via the PERK-Nrf2 signaling pathway [31]. Based on this evidence, our study focused on whether the transplantation of PRL-1-overexpressing PD-MSCs suppressed ER stress-dependent calcium influx in the livers of a rat model of cirrhosis induced by BDL, whether these cells had a positive effect, and elucidation of the mechanisms is involved. 


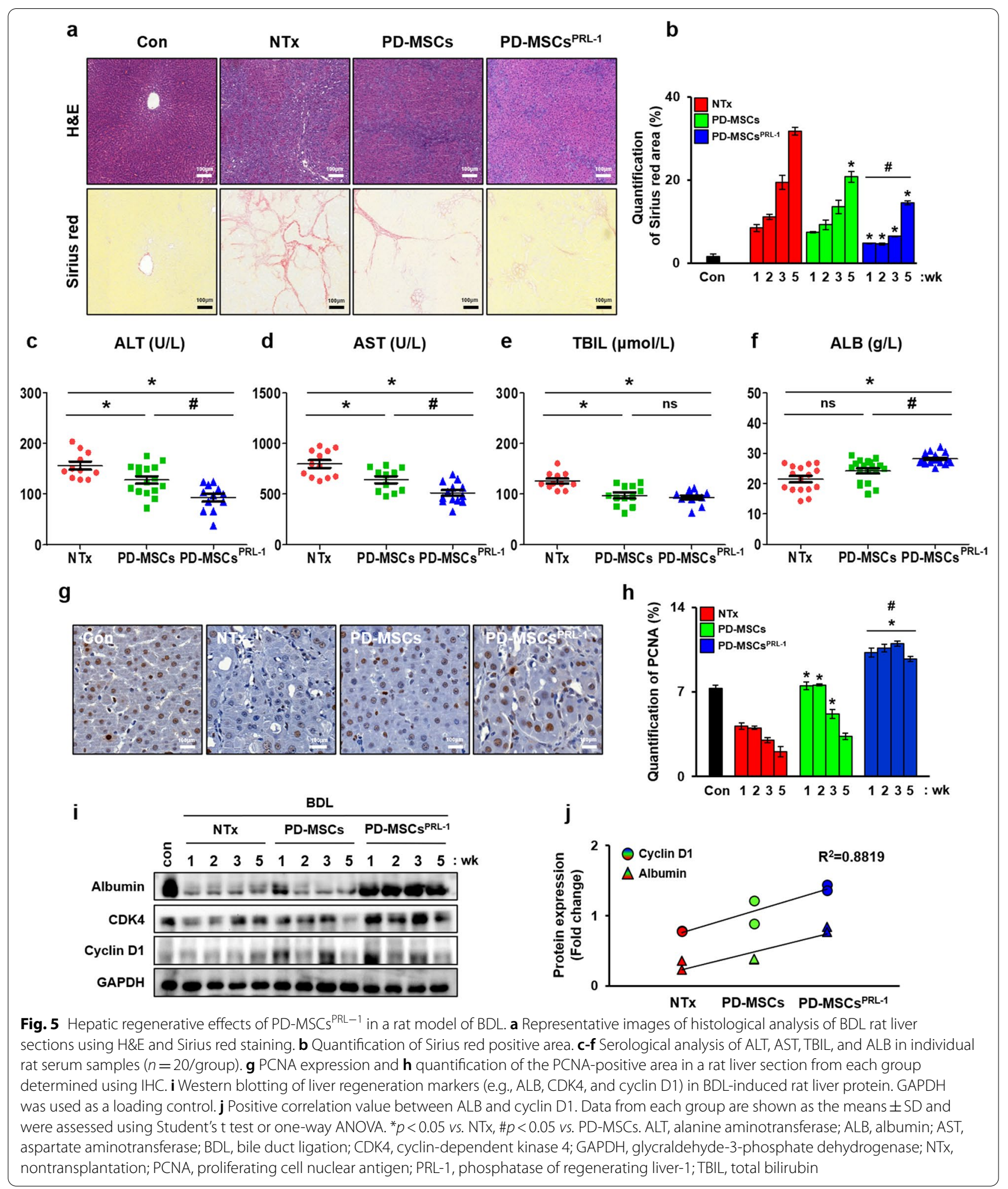

The expression of $\alpha$-SMA and transforming growth factor 1 and the number of apoptotic and necrotic hepatocytes were decreased in CHOP knockout mice following BDL-induced cirrhosis, which resulted in an ER stress response. Therefore, an increased ER stress response induced the overexpression of $\mathrm{CHOP}$ in bile 


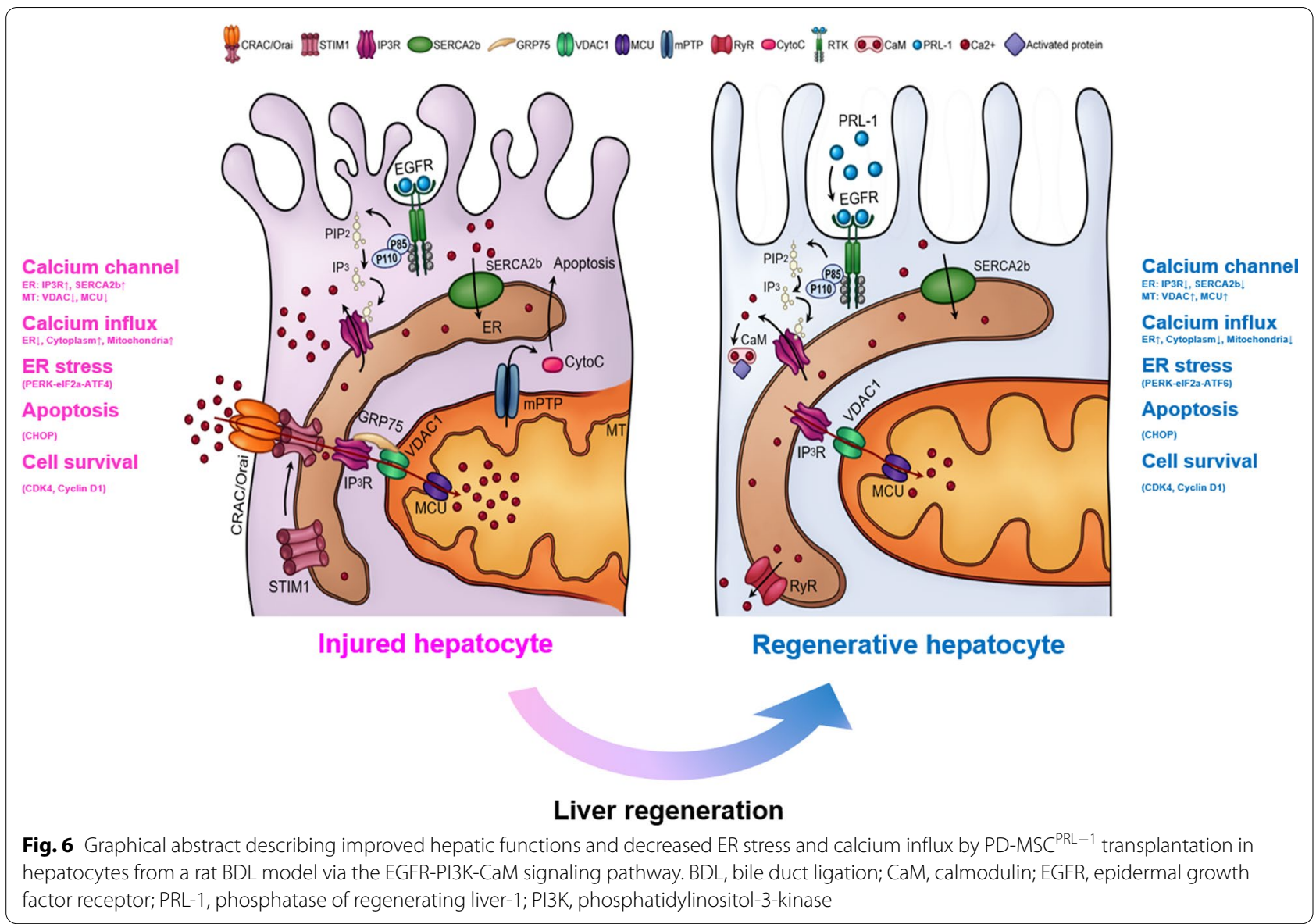

acid-induced hepatocytes via PERK-eIF2 $\alpha$-ATF4 signaling [7]. BM-MSC transplantation in high-fat diet (HFD)induced rats with NAFLD alleviated ATF4 and CHOP expression [32]. MSC treatment in palmitic acid-induced hepatocytes also reduced CHOP expression, and SERCA2b silencing reversed the ER stress response and calcium homeostasis [33]. These data are similar to our data, which showed that CHOP expression and PERK-eIF2 $\alpha-$ ATF4 signaling were increased in the BDL-injured rat model (Fig. 1). We observed changes in calcium channel factors in ER membranes (e.g., SERCA2b and IP3R) and mitochondrial membranes (e.g., VDAC1 and $\mathrm{MCU}$ ) in BDL-induced rat livers. Following PD-MSC or PDMSC $^{\text {PRL-1 }}$ transplantation, the levels of ER stress-related markers and calcium channel markers were dramatically reduced in a rat model of BDL. Treatment of hepatocytes with the SERCA inhibitor TG induced calcium depletion in the ER and the ER stress response via enhancement of the PERK-eIF2 $\alpha$-ATF4-CHOP axis and calcium channel markers in mitochondria. Compared to naïve PDMSCs, PD-MSCs ${ }^{\mathrm{PRL}-1}$ produced dramatic changes in ER $\mathrm{Ca}^{2+}$ transport factors in vivo and in vitro (Figs. 1, 2, 3). These results suggest that activated nonparenchymal cells (e.g., Kupffer cells and hepatic stellate cells) and parenchymal cells (e.g., hepatocytes) in a chronic liver disease model were different in the ER stress response and cell death injury [34]. Glucose-regulated protein 78 (GRP78) knockdown alleviated ER stress in activated LX2 cells and restored intracellular calcium levels via the overexpression of SERCA2 [35]. Many reports indicated that treatment of hepatocytes with several bile acids increased the expression of ER stress-related genes (e.g., Bip, XBP1, and $\mathrm{CHOP}$ ), calcium release and mitochondrial oxidative stress [36]. Bile acid accumulation also activated GRP78 and X-box binding protein 1 (XBP1) [37].

The present study confirmed a significant difference in the expression of PD-MSCs ${ }^{\mathrm{PRL}-1}$ compared to naïve PD-MSCs after treatment with $100 \mu \mathrm{M}$ LCA and cocultured with WB-F344 cells. In contrast to previous results, the expression pattern was similar to after treatment with TG and LCA in WB-F344 cells (Additional file 1: Figures S5, S6). Calcium influx was analyzed in TG-treated hepatocytes using a genetically encoded $\mathrm{Ca}^{2+}$ biosensor in the ER, mitochondria, and cytoplasm. PD-MSC ${ }^{\text {PRL-1 }}$ cocultivation resulted in dynamic changes and recovered calcium levels in the ER (Fig. 3). 
However, hepatocyte-specific calcium channels in the ER and mitochondria must be further confirmed for isolation from the ER and mitochondria in hepatocytes. Our previous reports demonstrated that the secreted PRL-1 level was significantly higher than naïve cells, functionenhanced PD-MSCs ${ }^{\mathrm{PRL}-1}$ were generated [38] and transplantation improved mitochondrial biogenesis and liver function in a BDL-injured rat model. A dramatic increase in the expression of exogenous PRL-1 was confirmed in the transplanted group with PD-MSCs ${ }^{\mathrm{PRL}-1}$ and siRNA treated in cocultured rat hepatocytes, and the expression was significantly decreased in PD-MSCs ${ }^{\mathrm{PRL}-1}$ compared to naive PD-MSCs. [20]. PD-MSCs ${ }^{\mathrm{PRL}-1}$ promoted antiadipogenic effects via improved IGFBP expression in orbital fibroblasts [39]. Accumulating evidence suggests that excessive intracellular lipids lead to deleterious ER stress and mitochondrial oxidative stress in hepatocytes [40].

Notably, we found that PD-MSCs ${ }^{\mathrm{PRL}-1}$ reduced ER stress via PERK-ATF4-CHOP signaling in the BDLinjured rat model and regulated the calcium imbalance between the ER and mitochondria, which resulted in improvements in hepatic function. PERK-dependent eIF2 $\alpha$ expression reduced the synthesis of cyclin D1 in stressed cells [41]. To further analyze the PRL-1-induced changes in calcium channels, we performed recombinant PRL-1 treatment and found that it increased IP3R, VDAC1, MCU, and GRP75 expression in LCA-induced hepatocytes (Additional file 1: Figure S7). We also found that PD-MSC ${ }^{\mathrm{PRL}-1}$ transplantation improved CDK4 and cyclin D1 expression, which led to the increased proliferation of hepatocytes compared to PD-MSCs (Fig. 5). These data are consistent with Suzuki et al. who reported that PRL-1 inhibited apoptosis with transactivation domains of the p53 transcriptional activator [17]. Following activation of the ER stress response, these molecules localized to the ER in a cell cycle-dependent manner and modulated mitosis [16]. PTPs, including PRL-1, contribute to calcium influx. PTPs are responsible for dephosphorylating EGFR, which indicates a similar specific activity as its Ser/Thr phosphatase activity [42]. Activated EGFR generates $\mathrm{Ca}^{2+}$ signals and subsequently controls the $\mathrm{Ca}^{2+} / \mathrm{CaM}$ complex [43]. Based on this evidence, we hypothesized that PRL-1 bound to the first signaling RTKs and affected intracellular calcium levels. Activated EGFR regulates ER stress-dependent calcium signaling, which activates phospholipase $\mathrm{C}$ gamma (PLCY) and the MAPK pathway [44]. We confirmed that phosphorylated EGFR expression was upregulated by recombinant PRL-1 or PD-MSCs ${ }^{\text {PRL-1 }}$ and activated the expression of the downstream factor PI3K-p110 $\alpha$, but not PI3K-p85, in the PD-MSC ${ }^{\mathrm{PRL}-1}$ groups (Fig. 4, Additional file 1: Figure S4). PD-MSC ${ }^{\mathrm{PRL}-1}$ transplantation suppressed $\mathrm{CaM}$ expression in hepatocytes, which resulted in decreased ER stress and calcium pathway activation. In briefly, we conclude that PRL-1 acts as a cellular trigger in regenerative hepatocytes by binding to the EGFR receptor, which alleviated ER stress and apoptosis and regulated the calcium imbalance between the ER and mitochondria. However, the fundamental mechanism between PRL-1 and EGFR requires further study.

\section{Conclusion}

The administration of $\mathrm{PD}-\mathrm{MSCs} \mathrm{sRL}^{\mathrm{PR}-1}$ decreased ER stress and modulated calcium levels via the EGFR-PI3KCaM signaling pathway, which led to improved hepatic functions in a rat model of BDL. Therefore, our data on this therapeutic mechanism are useful for the development of next-generation MSC-based stem cell therapy for regenerative medicine in chronic liver disease.

\section{Abbreviations}

ALT: Alanine aminotransferase; ALB: Albumin; a-MEM: Alpha-modified minimal essential medium; AST: Aspartate aminotransferase; ATF4: Activating transcription factor 4; BDL: Bile duct ligation; CaM: Calmodulin; CDK4: Cyclindependent kinase 4; CHOP: C/EBP homologous protein; DAPI: 4',6-Diamidino2-phenylindole; EGFR: Epidermal growth factor receptor; FBS: Fetal bovine serum; GAPDH: Glyceraldehyde 3-phosphate dehydrogenase; GFP: Green fluorescent protein; GRP75: Glucose regulated protein 75; GRP78: Glucose regulated protein 78; HFD: High-fat diet; hFGF-4: Human fibroblast growth factor-4; H\&E: Hematoxylin \& eosin; IP3R: Inositol trisphosphate receptor; LCA: Lithocholic acid; MCU: Mitochondria calcium uniporter; NBF: Neutral buffered formalin; NTx: Nontransplantation; PERK: PKR-like ER kinase; PCNA: Proliferating cell nuclear antigen; PD-MSCs: Placenta-derived mesenchymal stem cells; p-elF2a: Phosphorylated elF2 alpha; PI3K: Phosphatidylinositol-3-kinase; PRL-1: Phosphatase of regenerating liver-1; P/S: Penicillin/streptomycin; RTK: Receptor tyrosine kinases; SERCA2b: Sarco/endoplasmic reticulum $\mathrm{Ca}^{2+}$ ATPase; SD: Sprague-Dawley; STIM1: Stromal interaction molecule 1; TBIL: Total bilirubin; TG: Thapsigargin; VDAC1: Voltage-dependent anion channel 1; XBP-1: X-box binding protein 1.

\section{Supplementary Information}

The online version contains supplementary material available at https://doi. org/10.1186/s13287-021-02616-y.

Additional file 1. Supplementary Fig. 1 Optimal concentration of thapsigargin (TG) in WB-F344. a TG was treated with each concentration $(100,500$, and $1000 \mathrm{nM})$ for $6,12,24$, and $48 \mathrm{~h}$ in WB-F344. XBP1 splice form was transformed upon TG $24 \mathrm{~h}$ treatment. GAPDH was used as loading control. GAPDH, glycraldehyde-3-phosphate dehydrogenase; TG, tapsigargin. Supplementary Fig. 2 PD-MSCs ${ }^{\text {PRL-1 }}$ regulated calcium channels in a rat BDL and hepatocyte treated with $\mathrm{TG}$. $\mathbf{a}-\mathbf{d}$ mRNA levels of calcium channels (e.g., IP3R, GRP75, VDAC1, and MCU) in a rat model with BDL. e-i mRNA levels of calcium channels (e.g., IP3R, GRP75, VDAC1, MCU, and CaM) exposed to TG (500 nM) for $24 \mathrm{~h}$ in WB-F344 by qRT-PCR. Data from each group are shown as the means \pm SD and were assessed using Student's t-test. ${ }^{*} \mathrm{p}<0.05$ vs. NTx, \#p $<0.05$ vs. PD-MSCs. BDL, bile duct ligation; CaM, calmodulin; GRP75, glucose regulated protein 75; IP3R, inositol trisphosphate receptor; MCU, mitochondria calcium uniporter; NTx, nontransplantation; PRL-1, phosphatase of regenerating liver-1; TG, thapsigargin; VDAC1, voltage dependent anion channel 1. Supplementary Fig. 3 Increased expression of SERCA2b and STIM1 in PD-MSCs ${ }^{\text {PRL-1 }}$ groups in a rat model with BDL. $\mathbf{a}, \mathbf{b}$ mRNA levels of ER-specific $\mathrm{Ca}^{2+}$ channel factor SERCA2b and $\mathrm{Ca}^{2+}$ sensor factor STIM1 in a rat model with BDL. Data from each group are shown as the means \pm SD and were assessed using 
Student's t-test. ${ }^{*} p<0.05$ vs. NTx, \#p $<0.05$ vs. PD-MSCs. BDL, bile duct ligation; NTx, nontransplantation; SERCA2b, sarco/endoplasmic reticulum $\mathrm{Ca}^{2+}$ ATPase; PRL-1, phosphatase of regenerating liver-1; STIM1, stromal interaction molecule 1. Supplementary Fig. 4 PRL-1 regulates EGFRPI3K-CaM calcium signaling in a BDL-injured rat liver and rat hepatocyte treated LCA. a mRNA levels of CaM and $\mathbf{b}$ PI3K-p85 in a rat model with BDL. c-e mRNA levels of intracellular calcium signaling (e.g., EGFR, PI3K, and CaM) induced LCA $(100 \mu \mathrm{M})$ and treated recombinant PRL-1 (rePRL-1; $500 \mathrm{pg}$ ) in WB-F344. Data from each group are shown as the means \pm SD and were assessed using Student's t-test. ${ }^{*} p<0.05$ vs. NTx, \#p $<0.05$ vs. PD-MSCs. BDL, bile duct ligation; CaM, calmodulin; EGFR, epidermal growth factor receptor; NTX, nontransplantation; PI3K, phosphatidylinositol-3-kinase. Supplementary Fig. 5 PD-MSCS ${ }^{\text {PLL-1 }}$ modulated calcium channels in a rat hepatocyte treated with LCA by qRT-PCR. a-g mRNA levels related to intracellular calcium transport channels factors (e.g., SERCA2b, IP3R, VDAC1, MCU, GRP75, CaM, and STIM1) induced LCA (100 $\mu M$ ) and co-cultivation with naïve PD-MSCs or PD-MSC $s^{\text {PRL-1 }}$ in WB-F344. Data from each group are shown as the means \pm SD and were assessed using Student's t-test. ${ }^{*} p<0.05$ vs. LCA $(100 \mu M)$, \#p < 0.05 vs. PD-MSCs. CaM, calmodulin; GRP75, glucose regulated protein 75; IP3R, inositol trisphosphate receptor; LCA, lithocholic acid; MCU, mitochondria calcium uniporter; PRL-1, phosphatase of regenerating liver-1; SERCA2b, sarco/ endoplasmic reticulum $\mathrm{Ca}^{2+}$ ATPase; STIM1, stromal interaction molecule 1; VDAC1, voltage dependent anion channel 1. Supplementary Fig. 6 PD-MSCs ${ }^{\text {PRL-1 }}$ regulated calcium channels in a rat hepatocyte treated with LCA and TG by western blotting. a Protein expression and $\mathbf{b}-\mathbf{f}$ their intensities of intracellular calcium transport channels factors (e.g., SERCA2b, IP3R, VDAC1, MCU, and GRP75) induced LCA (100 $\mu \mathrm{M})$ and co-cultivation with naïve PD-MSCs or PD-MSCS ${ }^{\mathrm{PLL}-1}$ in WB-F344. $g$ Protein expression and h-j their intensities of intracellular calcium transport channels factors (e.g., SERCA2b, and MCU) and liver regeneration factor induced LCA (100 $\mu \mathrm{M})$ and co-cultivation with naïve PD-MSCs or PD-MSCs ${ }^{\text {PRL-1 }}$ and Pentamidine $(10 \mu \mathrm{g})$ treated in rat primary hepatocytes. GAPDH and a-tubulin was used as loading control. Data from each group are shown as the means \pm SD and assessed using Student's t-test. ${ }^{*} p<0.05$ vs. LCA $(100 \mu M)$, \#p $<0.05$ vs. PD-MSCs, $\$ p<0.05$ vs. PD-MSCs or PD-MSCs ${ }^{\text {PRL-1 }}$. ALB, Albumin; GAPDH, glycraldehyde-3-phosphate dehydrogenase; GRP75, glucose regulated protein 75; IP3R, inositol trisphosphate receptor; LCA, lithocholic acid; MCU, mitochondria calcium uniporter; PRL-1, phosphatase of regenerating liver-1; SERCA2b, sarco/endoplasmic reticulum $\mathrm{Ca}^{2+}$ ATPase; TG, Thapsigargin; VDAC1, voltage dependent anion channel 1. Supplementary Fig. 7 Recombinant PRL-1 increased calcium channels in a rat hepatocyte treated with LCA. a-g mRNA levels of intracellular calcium transport channels factors (e.g., SERCA2b, IP3R, VDAC1, MCU, GRP75, CaM, and STIM1) induced LCA (100 $\mu \mathrm{M})$ and treated recombinant PRL-1 (rePRL-1; $500 \mathrm{pg}$ ) in WB-F344. Data from each group are shown as the means \pm SD and assessed using Student's t-test. ${ }^{*} p<0.05$ vs. LCA (100 $\mu M)$. CaM, calmodulin; GRP75, glucose regulated protein 75; IP3R, inositol trisphosphate receptor; LCA, lithocholic acid; MCU, mitochondria calcium uniporter; PRL-1, phosphatase of regenerating liver-1; SERCA2b, sarco/ endoplasmic reticulum $\mathrm{Ca}^{2+}$ ATPase; VDAC1, voltage dependent anion channel 1 .

\section{Acknowledgements}

We thank Ho Jeong Kim (Incheon Catholic University) for helping us with the graphical abstract.

\section{Authors' contributions}

SHK and JYK did analysis and interpretation of data and manuscript drafting. SHK, JYK, and WTJ did animal model construction, validation, and formal analysis. SHK, JYK, and SYP helped critical comments. JMK and SHB did project administration and funding acquisition. GJK conceived and designed the experiments and contributed to the manuscript drafting and final approval of manuscript. All authors have read and agreed to the published version of the manuscript.

\section{Funding}

This research was supported by a grant of the Korea Health Technology R\&D Project through the Korea Health Industry Development Institute (KHIDI), funded by the Ministry of Health \& Welfare, Republic of Korea (H117C1050) and Basic Science Research Program through the National Research Foundation of Korea (NRF) funded by the Ministry of Education, Science and Technology (2020M3A9B302618221).

\section{Availability of data and materials}

The data that support the findings of this study are available from the corresponding author upon reasonable request.

\section{Declarations}

\section{Ethics approval and consent to participate}

The collection of samples and their use for research purposes were approved by the institutional Review Board of CHA Bundang Hospital, Seoul, Republic of Korea (IRB 07-18). All participants provided written informed consent prior to sample collection. The experimental processes and protocols for animal modeling were approved by the Institutional Animal Care Use Committee of CHA University, Seongnam, Republic of Korea (IACUC-200033).

\section{Consent for publication}

Not applicable.

\section{Competing interests}

The authors declare that there has no conflict of interest.

\section{Author details}

${ }^{1}$ Department of Biomedical Science, CHA University, Seongnam 13488, Republic of Korea. ${ }^{2}$ Research Institute of Placenta Science, CHA University, Seongnam 13488, Republic of Korea. ${ }^{3}$ Department of Oral Microbiology and Immunology, School of Dentistry and Dental Research Institute, Seoul National University, Seoul 08826, Republic of Korea. ${ }^{4}$ Department of Internal Medicine, Catholic University Medical College, Seoul 06591, Republic of Korea.

Received: 12 July 2021 Accepted: 6 October 2021

Published online: 24 October 2021

\section{References}

1. Malhi H, Kaufman RJ. Endoplasmic reticulum stress in liver disease. J Hepatol. 2011;54(4):795-809.

2. Di Conza G, Ho PC. ER Stress Responses: An Emerging Modulator for Innate Immunity. Cells. 2020;9(3).

3. Maiers JL, Malhi H. Endoplasmic Reticulum Stress in Metabolic Liver Diseases and Hepatic Fibrosis. Semin Liver Dis. 2019;39(2):235-48.

4. Kao E, Shinohara M, Feng M, Lau MY, Ji C. Human immunodeficiency virus protease inhibitors modulate $\mathrm{Ca} 2+$ homeostasis and potentiate alcoholic stress and injury in mice and primary mouse and human hepatocytes. Hepatology. 2012;56(2):594-604.

5. Jousset H, Frieden M, Demaurex N. STIM1 knockdown reveals that store-operated Ca2+ channels located close to sarco/endoplasmic Ca2+ ATPases (SERCA) pumps silently refill the endoplasmic reticulum. J Biol Chem. 2007;282(15):11456-64.

6. Ni HM, Baty CJ, Li N, Ding WX, Gao W, Li M, Chen X, Ma J, Michalopoulos GK, Yin XM. Bid agonist regulates murine hepatocyte proliferation by controlling endoplasmic reticulum calcium homeostasis. Hepatology. 2010:52(1):338-48.

7. Tamaki N, Hatano E, Taura K, Tada M, Kodama Y, Nitta T, Iwaisako K, Seo S, Nakajima A, Ikai I, Uemoto S. CHOP deficiency attenuates cholestasisinduced liver fibrosis by reduction of hepatocyte injury. Am J Physiol Gastrointest Liver Physiol. 2008;294(2):G498-505.

8. Lemmon MA, Schlessinger J. Cell signaling by receptor tyrosine kinases. Cell. 2010;141(7):1117-34.

9. Berasain C, Avila MA. The EGFR signalling system in the liver: from hepatoprotection to hepatocarcinogenesis. J Gastroenterol. 2014;49(1):9-23.

10. Komposch K, Sibilia M. EGFR Signaling in Liver Diseases. Int J Mol Sci. 2015;17(1).

11. Wang Y, Li G, Goode J, Paz JC, Ouyang K, Screaton R, Fischer WH, Chen J, Tabas I, Montminy M. Inositol-1,4,5-trisphosphate receptor 
regulates hepatic gluconeogenesis in fasting and diabetes. Nature. 2012;485(7396):128-32.

12. Ozcan U, Cao Q, Yilmaz E, Lee AH, Iwakoshi NN, Ozdelen E, Tuncman G, Gorgun C, Glimcher LH, Hotamisligil GS. Endoplasmic reticulum stress links obesity, insulin action, and type 2 diabetes. Science. 2004;306(5695):457-61.

13. Wei M, Korotkov KV, Blackburn JS. Targeting phosphatases of regenerating liver (PRLs) in cancer. Pharmacol Ther. 2018;190:128-38.

14. Kim JY, Jun JH, Park SY, Yang SW, Bae SH, Kim GJ. Dynamic Regulation of miRNA Expression by Functionally Enhanced Placental Mesenchymal Stem Cells PromotesHepatic Regeneration in a Rat Model with Bile Duct Ligation. Int J Mol Sci. 2019;20(21).

15. Boverhof DR, Burgoon LD, Tashiro C, Sharratt B, Chittim B, Harkema JR, Mendrick DL, Zacharewski TR. Comparative toxicogenomic analysis of the hepatotoxic effects of TCDD in Sprague Dawley rats and C57BL/6 mice. Toxicol Sci. 2006;94(2):398-416.

16. Wang J, Kirby CE, Herbst R. The tyrosine phosphatase PRL-1 localizes to the endoplasmic reticulum and the mitotic spindle and is required for normal mitosis. J Biol Chem. 2002;277(48):46659-68.

17. Suzuki S, Tsutsumi S, Chen Y, Ozeki C, Okabe A, Kawase T, Aburatani $\mathrm{H}_{\text {, }}$ Ohki R. Identification and characterization of the binding sequences and target genes of p53 lacking the 1st transactivation domain. Cancer Sci. 2020;111(2):451-66.

18. He J, Yao J, Sheng H, Zhu J. Involvement of the dual-specificity tyrosine phosphorylation-regulated kinase 1A-alternative splicing factor-calcium/ calmodulin-dependent protein kinase Ildelta signaling pathway in myocardial infarction-induced heart failure of rats. J Card Fail. 2015;21(9):751-60.

19. Jung J, Choi JH, Lee Y, Park JW, Oh IH, Hwang SG, Kim KS, Kim GJ. Human placenta-derived mesenchymal stem cells promote hepatic regeneration in CCl4 -injured rat liver model via increased autophagic mechanism. Stem Cells. 2013;31(8):1584-96.

20. Kim JY, Choi JH, Jun JH, Park S, Jung J, Bae SH, Kim GJ. Enhanced PRL-1 expression in placenta-derived mesenchymal stem cells accelerates hepatic function via mitochondrial dynamics in a cirrhotic rat model. Stem Cell Res Ther. 2020;1 1(1):512.

21. Lee MJ, Jung J, Na KH, Moon JS, Lee HJ, Kim JH, Kim Gl, Kwon SW, Hwang SG, Kim GJ. Anti-fibrotic effect of chorionic plate-derived mesenchymal stem cells isolated from human placenta in a rat model of CCI(4)-injured liver: potential application to the treatment of hepatic diseases. J Cell Biochem. 2010;111(6):1453-63.

22. Kountouras J, Billing BH, Scheuer PJ. Prolonged bile duct obstruction: a new experimental model for cirrhosis in the rat. Br J Exp Pathol. 1984;65(3):305-11.

23. Li D, Wang WJ, Wang YZ, Wang YB, Li YL. Lobaplatin promotes (125) I-induced apoptosis and inhibition of proliferation in hepatocellular carcinoma by upregulating PERK-elF2al pha-ATF4-CHOP pathway. Cell Death Dis. 2019;10(10):744.

24. Arruda AP, Pers BM, Parlakgul G, Guney E, Inouye K, Hotamisligil GS. Chronic enrichment of hepatic endoplasmic reticulum-mitochondria contact leads to mitochondrial dysfunction in obesity. Nat Med. 2014;20(12):1427-35.

25. Ali ES, Petrovsky N. Calcium Signaling As a Therapeutic Target for Liver Steatosis. Trends Endocrinol Metab. 2019;30(4):270-81.

26. Bahar E, Kim H, Yoon H. ER Stress-Mediated Signaling: Action Potential and $\mathrm{Ca}(2+)$ as Key Players. Int J Mol Sci. 2016;17(9).

27. Jo H, Choe SS, Shin KC, Jang H, Lee JH, Seong JK, Back SH, Kim JB. Endoplasmic reticulum stress induces hepatic steatosis via increased expression of the hepatic very low-density lipoprotein receptor. Hepatology. 2013;57(4):1366-77.

28. Li K, Xiao Y, Yu J, Xia T, Liu B, Guo Y, Deng J, Chen S, Wang C, Guo F. Liverspecific Gene Inactivation of the Transcription Factor ATF4 Alleviates Alcoholic Liver Steatosis in Mice. J Biol Chem. 2016;291(35):18536-46.

29. Wang X, Zhuang Y, Fang Y, Cao H, Zhang C, Xing C, Guo X, Li G, Liu P, Hu G, Yang F. Endoplasmic reticulum stress aggravates copper-induced apoptosis via the PERK/ATF4/CHOP signaling pathway in duck renal tubular epithelial cells. Environ Pollut. 2021;272:115981.
30. Chen Y, Zhang F, Wang D, Li L, Si H, Wang C, Liu J, Chen Y, Cheng J, Lu Y. Mesenchymal Stem Cells Attenuate Diabetic Lung Fibrosis via Adjusting Sirt3-Mediated Stress Responses in Rats. Oxid Med Cell Longev. 2020;2020:8076105.

31. Lee EJ, Cardenes N, Alvarez D, Sellares J, Sembrat J, Aranda P, Peng Y, Bullock J, Nouraie SM, Mora AL, Rojas M. Mesenchymal stem cells reduce ER stress via PERK-Nrf2 pathway in an aged mouse model. Respirology. 2020;25(4):417-26.

32. Ulum B, Teker HT, Sarikaya A, Balta G, Kuskonmaz B, Uckan-Cetinkaya D, Aerts-Kaya F. Bone marrow mesenchymal stem cell donors with a high body mass index display elevated endoplasmic reticulum stress and are functionally impaired. J Cell Physiol. 2018;233(11):8429-36.

33. Li L, Zeng X, Liu Z, Chen X, Li L, Luo R, Liu X, Zhang J, Liu J, Lu Y, et al. Mesenchymal stromal cells protect hepatocytes from lipotoxicity through alleviation of endoplasmic reticulum stress by restoring SERCA activity. J Cell Mol Med. 2021;25(6):2976-93.

34. Hu J, Han H, Lau MY, Lee H, MacVeigh-Aloni M, Ji C. Effects of combined alcohol and anti-HIV drugs on cellular stress responses in primary hepatocytes and hepatic stellate and kupffer cells. Alcohol Clin Exp Res. 2015;39(1):11-20.

35. Wang H, Han B, Wang N, Lu Y, Gao T, Qu Z, Yang H, Yang Q. Oxymatrine attenuates arsenic-induced endoplasmic reticulum stress and calcium dyshomeostasis in hepatic stellate cells. Ann Transl Med. 2020;8(18):1171.

36. Cai SY, Ouyang X, Chen Y, Soroka CJ, Wang J, Mennone A, Wang Y, Mehal WZ, Jain D, Boyer JL. Bile acids initiate cholestatic liver injury by triggering a hepatocyte-specific inflammatory response. JCI Insight. 2017;2(5):e90780

37. Bochkis IM, Rubins NE, White P, Furth EE, Friedman JR, Kaestner KH. Hepatocyte-specific ablation of Foxa2 alters bile acid homeostasis and results in endoplasmic reticulum stress. Nat Med. 2008;14(8):828-36.

38. Kim JY, Choi JH, Kim SH, Park H, Lee D, Kim GJ. Efficacy of Gene Modification in Placenta-Derived Mesenchymal Stem Cells Based on Nonviral Electroporation. Int J Stem Cells. 2021;14(1):112-8.

39. Kim JY, Park S, Lee HJ, Lew H, Kim GJ. Functionally enhanced placentaderived mesenchymal stem cells inhibit adipogenesis in orbital fibroblasts with Graves' ophthalmopathy. Stem Cell Res Ther. 2020;11(1):469.

40. Zhang X, Zhang K. Endoplasmic Reticulum Stress-Associated Lipid Droplet Formation and Type II Diabetes. Biochem Res Int. 2012;2012:247275.

41. Brewer JW, Diehl JA. PERK mediates cell-cycle exit during the mammalian unfolded protein response. Proc Natl Acad Sci U S A. 2000;97(23):12625-30.

42. Pallen CJ, Valentine KA, Wang $\mathrm{JH}$, Hollenberg MD. Calcineurin-mediated dephosphorylation of the human placental membrane receptor for epidermal growth factor urogastrone. Biochemistry. 1985;24(18):4727-30.

43. Sanchez-Gonzalez P, Jellali K, Villalobo A. Calmodulin-mediated regulation of the epidermal growth factor receptor. FEBS J. 2010;277(2):327-42.

44. Rachakhom W, Khaw-On P, Pompimon W, Banjerdpongchai R. Dihydrochalcone Derivative Induces Breast Cancer Cell Apoptosis via Intrinsic, Extrinsic, and ER Stress Pathways but Abolishes EGFR/MAPK Pathway. Biomed Res Int. 2019;2019:7298539.

\section{Publisher's Note}

Springer Nature remains neutral with regard to jurisdictional claims in published maps and institutional affiliations.

Ready to submit your research? Choose BMC and benefit from:

- fast, convenient online submission

- thorough peer review by experienced researchers in your field

- rapid publication on acceptance

- support for research data, including large and complex data types

- gold Open Access which fosters wider collaboration and increased citations

- maximum visibility for your research: over $100 \mathrm{M}$ website views per year

At BMC, research is always in progress.

Learn more biomedcentral.com/submissions 